\title{
Ward round documentation in a major trauma centre: can we improve patient safety?
}

Gemma Green, Arash Aframian, jason bernard

St George's Hospital NHS Trust, UK

\begin{abstract}
Our objective was to improve documentation and patient safety in a major trauma centre.

A retrospective audit was undertaken in March 2014. Ward round entries for each orthopaedic patients on three dates were assessed against standards and analysed. The audit was repeated in April 2014, and again in August 2014.

Thorough documentation is paramount in a major trauma centre. It forms a useful record of the patients hospital stay, is a legal document and is highlighted in national guidelines. It provides a basis for good handover, ensuring continuation of care and maintaining patient safety. Resultant poor compliance with Royal College guidelines in the initial audit led to the production of a new electronic based note keeping system. A meeting was held with all staff prior to introduction.

Our initial results gained 75 entries, and none showed full compliance. Mean compliance per entry was 59\% (0-81\%). The second attempt gained 90 entries, with 30 from the weekend. Mean compliance per entry 97\%. Third attempt received 61 entries, with 27 from the weekend. Mean compliance was $96 \%$, meaning that the improvement was being maintained.
\end{abstract}

Recent distressing reports regarding patient highlighted the importance of patient. Our initial audit proved there were many areas lacking in our documentation and improvement was necessary. Prior to introducing electronic systems, the implemented change has produced improvement in documentation, and provides a useful handover tool for staff.

\section{Problem}

Patient safety is of paramount importance in the provision of good medical care, and is highlighted in the recent Francis enquiry.[1] In busy major trauma centres such as St George's Hospital, maintaining clinical and professional standards through the utilisation of robust care pathways, policies and procedures is essential in avoiding patient safety issues in chaotic circumstances. Given the current shift-based system worked by many juniors, as well as the drive to increasing use of advanced nurse practitioners and physician associates (as in our institution), the quality documentation of the patients stay is important to achieve good handover, continuity of care, and ultimately patient safety.[2]

Medical notes are considered to be a legal document, and therefore should be legible, contemporaneous, and kept in a place of safety. In our busy ward environment, a "consultant-of-the-week" system is employed for trauma. Ward rounds are carried out daily of all inpatients; the number of which can range from 50-90 inpatients, necessitating a rapid and efficient process.[3] At a clinical governance meeting in February 2014, concerns were raised about the quality of ward rounds and the documentation performed on these. The poor documentation led to confusion regarding patient care, and therefore impacted on junior staff, nursing staff, and more importantly the patients themselves.

\section{Background}

Clinical governance issues, such as documentation, are highly topical in current literature. Papers suggest that good documentation provides a basis for excellent handover, and ensures completeness and coherence to the patient pathway.[2-6] Ward rounds are have been shown to be useful clinical experiences that have many uses: establishing diagnoses, making management plans, formulating discharge procedures, and as a means of providing safety by checking prescription charts.[3] Orthopaedic ward rounds, like most specialty ward rounds, are complex and proceed in a rapid manner in order to provide care to many patients as well as organising theatre activities. In this rapid turnover specialty, efficient and comprehensive documentation is required.

There are several recognised obstacles to effective documentation in the literature, including coordinating multiple healthcare professionals, working within a unit with numerous consultants with different working styles, and the loss of the "firm" structure and ownership of patients.[7-9] All of these challenges to the running of ward rounds have a pronounced effect on the quality of documentation. Additionally, many junior team members have little training in the requirements for good documentation. Many centres are beginning to employ electronic documentation as this has been shown to be much safer and more reliable than written notes, however the systems are not yet in place at St George's in order for this to occur.[10] 


\section{Baseline measurement}

A retrospective audit of note keeping was undertaken in March 2013. Ward round entries for each orthopaedic patient on three dates were assessed according to recognised Royal College Standards and data were entered into an Excel spreadsheet and analysed.

The criteria required to be documented were: patient name, hospital number, location, time (including current/retrospective nature of notekeeping), consultant in charge of patient's care, diagnosis, current issues, early warning score, venous thromboprophylaxis, antibiotic prescription, discharge plan, and that the note was signed and a contact number or bleep given.

The target for the audit was set at 95\% compliance. The results obtained from the initial audit are shown in the table. The criterion with the highest compliance understandably was the patient's name, although even for this fundamental aspect, compliance was only $89 \%$. Only $4 \%$ of records were signed, and bleep numbers documented. Other poorly documented criteria included the antibiotic prescription and time of record.

The graph shows the consistency of entries, ie how may criteria are met per entry. The diffuse spread of results show that documentation is inconsistent

See supplementary file: ds4134.docx - "baseline results revised (1)"

\section{Design}

The Royal College of Surgeons, along with the Academy of Medical Royal Colleges, produce guidance on the content and frequency of documentation and ward round entries; this allows for specific and attainable criteria to measure against. It also aided the design of our new notes proforma. We created a spreadsheet that contained all the criteria required as subject headings. This allowed us to collect data and keep it up to date for each patient daily. Unfortunately, the only paper that we are permitted to use in our medical records is the St Georges NHS history sheet, so we mail-merged the data from our updated spreadsheet onto a word document that was formatted to fit to the standard history sheet paper.

This meant that the static information could then be printed daily, with the demographics, diagnosis, antibiotic prescription, and thromboembolic prophylaxis already inserted. The ward round consultant plan and any current issues could then be inserted on the round, saving time and ensuring a useful summary is provided each day. This also helps the continuity of patient care, as each member of the team has a summary of the patient's diagnosis, plan, and progress.

A meeting was held with all ward staff; this included the ward managers of each ward, the junior medical team, physician associates, and discharge coordinator. Discussions were had about the concerns raised over ward round etiquette and documentation, and the new documentation and ward round strategy outlined.
Response was unanimously positive and staff were keen to commence.

Alterations were made after a week's trial of the prototype spreadsheet and proforma (such as adding length of stay) and then the new system fully implemented. The plan was to audit at one month and six months post implementation in order to assess if improvement achieved, and if so whether this was maintained.

Due to the constant turnover of medical juniors it was key to include and inspire the permanent members of the team including the nurses and physician associates in order to ensure longevity of the project.

\section{Strategy}

The spreadsheet and proforma were shown to all ward staff and their opinions sought. Proformas were tailored in order to make them easy to use for all staff. As the use is on trauma ward rounds, all trauma consultants were made aware of the plan for a change to the documentation system. Formatting of the word document to fit onto a standard St George's history sheet posed several problems as the line size on NHS history paper is rather different from any format on Microsoft word. After several attempts the formatting was completed.

The new system was trialled for a week prior to its introduction in order to iron out any issues. Issues arose with new admissions not being added automatically to the spreadsheet, therefore on-call doctors were advised to input them as part of their handover.

At the second cycle in April there were several spelling mistakes and numerical typing errors identified. These were highlighted to the ward staff, but are beyond the scope of the question of the audit. The feeling after the second cycle was that the new system helped to structure the ward rounds and enabled all staff to know the patient diagnosis and plan.

The third cycle demonstrated that the system was continuing to be used, and that notes were more standardised. Staff continue to interact well with the system. The next strategy is to make the whole process electronic, but this is still early in its progression.

\section{Results}

In the initial audit there were 74 ward round entries assessed. As discussed in baseline results section, the compliance was poor, with a range of $4 \%-89 \%$ compliance with criteria, and an average compliance of $67 \%$. This was well below the standard set at $95 \%$.

The second cycle was undertaken in April 2014, one month after introduction; again using ward round entries from three non consecutive days over two weeks. Sixty entries were assessed, of which 26/60 were from weekend rounds. The cycle 2 table shows the percentage compliance one month following introduction of our new notes system. The average compliance has improved to $97 \%$ which is above the target set. The lowest compliance was seen to 
BMJ Quality Improvement Reports

be the signature of the member of staff. Half of the criteria scored $100 \%$. Using the students $T$ test there was a significant improvement in compliance after the second audit loop, $p=0.0012$. There was also a significant difference in the consistency of ward round entries (i.e the percentage compliance per entry) $p<0.00001$.

A third cycle was performed at six months after introduction using the same methods. Again, the loop 3 table show the percentage compliance per criterion. The average compliance was $96 \%$, remaining above the set target. The criterion with the lowest documentation was the discharge plan at $69 \%$, but nine out of the 12 criteria had been $100 \%$ achieved. There was no statistical difference using the student $\mathrm{T}$ test between the results from the second and third loops, $\mathrm{P}=0.203$.

See supplementary file: ds4133.docx - "results loop 2 and 3 revised"

\section{Lessons and limitations}

Many obstacles to improvement existed in the implementation of this quality improvement project, the largest of which was the rigidity of the system already in place and the attitude of "this is how we have always done it." By holding a meeting with ward staff, we were able to highlight each persons concerns about the old system and how the new system would address this. It also allowed a brainstorming session to occur in order to ensure all criteria were adequately covered, and also devise a more efficient, staff-friendly system of carrying out ward rounds.

Ultimately the aim is to achieve a paperless system of documentation at our trust, however the information technology systems, policies and infrastructure are still far from ready. Given this and the express nature of the trauma ward rounds, introduction of electronic notekeeping may have it's initial downfalls. In the interim, and this could be medium to long term, the new notes system provides a robust and easy to use system which fulfils criteria set out by Royal Colleges and therefore improves patient safety.

As all members of staff were involved in implementing the change, the system has longevity. In particular the permanent members of ward staff (ie the nurses and physician associates) were involved in defining their roles and were able to convey their needs and opinions. Our notes proforma has allowed the ward rounds to have more fluidity and structure, as both medical and nursing staff are guided by the structure of the proforma.

Several limitations are notable to this project. Firstly the data collected reflected only the presence of the documentation of individual criteria; this was not cross checked with charts and notes to ascertain whether all information was correct. Secondly, because the physician associates do not work at weekends and the staffing levels medically are very low, often patients admitted over the weekend had no entry on the spreadsheet on a Monday morning, and therefore written documentation for the Monday entry rather than the proforma was used. This appeared to improve at the six month point, when ward staff were more used to operating the new system. Finally there were frequent spelling errors on pre-printed sheets.

\section{Conclusion}

We show that our new system of note keeping for use during ward rounds in a major trauma centre improves both the quality and consistency of ward round entries. It consolidates each member of the team's knowledge about each patient, and contributes to effective handover. Until the introduction of fully electronic patient records this system provides a comprehensive way of documenting the patient pathway.

As the second loop was completed at one month (which is very early) it was decided that another audit cycle will be completed at six months post introduction in order to assess longevity. We have also started to assess staff satisfaction with the system and the impact on the running of ward rounds on the orthopaedic wards.

\section{References}

1. Robert Francis QC. The Mis Staffordshire NHS foundation Trust Public Enquiry 2013. Accessed at http://www.midstaffspublicinquiry.com/sites/default/files/repo rt/Executive\%20summary.pdf on 28/8/14 at 20:28

2. Al-Mahrouqi H., Oumer R, Tapper R, Roberts R. Post-acute surgical ward round proforma improves documentation. BMJ QIR 2013;2. http://qir.bmi.com/content/2/1/u201042.w688.full

3. NHS Services, Seven Days a Week Forum. Evidence based medicine. http://www.england.nhs.uk/wpcontent/uploads/2013/12/evidence-base.pdf

4. Till A, Sall H, Wilkinson J. Safe Handover, Safe Patientsthe electronic handover. BMJ QIR 2014. http://air.bmi.com/content/2/2/u202926.w1359.full? sid=8448 de6b-1d62-45cb-ad90-28a9f5512833

5. Mann R, Williams J. Standards in medical record keeping. Clinical Medicine, Journal of the Royal College of Physicians 2003. 3(4);329-32.

6. Good Medical Practice 2012. GMC guidance. http://www.gmc-uk.org/Good_Medical_Practice_2012_Dr aft for consultation.pdf 45081179.pdf

7. HSCIC and Academy of Medical Royal Colleges. Standards for the clinical structure and content of patient records. http://www.rcplondon.ac.uk/sites/default/files/standards-forthe-clinical-structure-and-content-of-patient-records.pdf

8. Good Surgical Practice 2012, Royal College of Surgeons. http://www.rcseng.ac.uk/surgeons/surgicalstandards/professionalism-surgery/good-surgical-practice

9. Ward rounds in medicine. Principles of best practice (2012). Accessed at http://www.rcplondon.ac.uk/sites/default/files/ward_rounds_i n medicine.pdf

10. BMA Safe Handover, Safe Patients. http://bma.org.uk/-/media/files/pdfs/practical\%20advice\%20 at\%20work/contracts/safe\%20handover\%20safe\%20patient s.pdf 


\section{BMJ Quality Improvement Reports}

11. Timm A (2013) the demise of the firm-what is happening to apprenticeship learning?

http://www.southampton.ac.uk/assets/imported/transforms/p eripheral-block/UsefulDownloads Download/4762464A4BA D4178B69F79EDB183F8ED/The\%20demise\%20of\%20the \%20firm Report v2.pdf

\section{Declaration of interests}

No conflicts of interest to declare.

\section{Acknowledgements}

I would like to acknowledge the hard working orthopaedic ward staff at St George's Hospital, including but not limited to: our physician's associates Miss Sam Cryer, Mr Dominic Pritchard and Mr Jonathan Ogidi. The senior nursing staff Ms Nicola Hicks, Ms Leslin Powell, Mr Tim Leak and Ms Karen Barrett, and the medical staff Mr Avtar Sur and Mr Mike Williamson. 